\title{
Proceeding
}

Supplementary Issue: Summer Conferences of Sports Science. Costa Blanca Sports Science Events, 25-26 September 2020. Alicante, Spain.

\section{Functional evaluation of the diabetic athlete: Role of the sports doctor}

\author{
VINCENZO CHRISTIAN FRANCAVILLA ${ }^{1}$, RITA POLITO2 $\triangle$, MARIA CHIARA PARISI ${ }^{1}$, OMAR GAETANO \\ MARIA MINGRINO ${ }^{1}$, ANGELO CAMPANOZZI ${ }^{3}$, AURORA DANIELE ${ }^{4}$, ANTONIETTA MESSINA ${ }^{5}$, \\ VINCENZO MONDA ${ }^{5}$, ANNA VALENZANO 6 \\ ${ }^{1}$ School of Engineering, Architecture, and Motor Sciences, Kore University of Enna, Enna, Italy \\ 2Department of Public Health, University of Naples "Federico II", Napoli, Italy \\ ${ }^{3}$ Pediatrics Department of Medical and Surgical Sciences, University of Foggia, Italy \\ ${ }^{4}$ Department of Biological and Pharmaceutical Environmental Sciences and Technologies, Luigi Vanvitelli University of Campania, \\ Naples, Italy \\ ${ }^{5}$ Department of Experimental Medicine, Section of Human Physiology and Unit of Dietetic and Sport Medicine, Luigi Vanvitelli \\ University of Campania, Naples, Italy \\ ${ }^{6}$ Department of Medical and Surgical Sciences, University of Foggia, Italy
}

\begin{abstract}
Obesity is a multifactorial disease and the most primary risk of this is the development of type 2 diabetes. Numerous studies show that the effects of obesity on glucose metabolism result in the deterioration of glucose tolerance, the development of insulin resistance and the consequent damage of the secretory function of beta-cells. The type 2 diabetes is a worldwide health problem associated with obesity and sedentary lifestyle. As reported the American Diabetes Association, the physical activity is fundamental for type 2 diabetes patients, reducing blood pressure, cardiovascular events, and improve glucose metabolism and insulin sensitivity. In the light of these evidences, the aim of this study is to analyse the effects of healthy lifestyle, characterized by a correct diet and by adequate physical activity program in type 2 diabetes patients. We analysed 25 sedentary type 2 diabetes patients subjected to a correct diet and therapeutic treatment and 20 active type 2 diabetes patients subjected to a correct nutrition and a program of physical activity. We found an amelioration in biochemical and anthropometric parameters such as blood pressure, glycemia, glycated haemoglobin, body mass index (BMI). Given these results we can conclude that the physical activity has always been considered one of the cornerstones of type 2 diabetes therapy together with diet and pharmacotherapy. However, the results obtained show that physical activity deserves the same level of consideration as diet and insulin therapy even in the subject with type 2 diabetes.

Keywords: Type 2 Diabetes; Obesity; Physical activity; Body mass index; Correct nutrition; Glycemia; Total cholesterol; Glycated haemoglobin.

Cite this article as:

Francavilla, V.C., Polito, R., Parisi, M.C., Mingrino, O.G.M., Campanozzi, A., Daniele, A., Messina, A., Monda, V., \& Valenzano, A. (2020). Functional evaluation of the diabetic athlete: Role of the sports doctor. Journal of Human Sport and Exercise, 15(4proc), S1344-S1353. doi:https://doi.org/10.14198/ihse.2020.15.Proc4.32

Corresponding author. Department of Public Health, University of Naples "Federico II", Via S. Pansini 5, 80131. Napoli, Italy. E-mail: rita.polito@unicampania.it

Abstract submitted to: Spring Conferences of Sports Science. Costa Blanca Sports Science Events, 19-20 June 2020. Alicante, Spain.

JOURNAL OF HUMAN SPORT \& EXERCISE ISSN 1988-5202

(c) Faculty of Education. University of Alicante

doi:10.14198/jhse.2020.15.Proc4.32
\end{abstract}

S1344

| 2020 | Proc4 | VOLUME 15

C 2020 University of Alicante 


\section{INTRODUCTION}

In the last decade, the attention of the Italian diabetological community has focused on the quality of life and therefore on a non-secondary aspect of physical and sports activity, thanks in part to the promotional impulse given by the Italian Association of Diabetic Athletes. Obesity is a multifactorial disease and the most primary risk of this is the development of type 2 diabetes. In addition, numerous studies show that the effects of obesity on glucose metabolism result in the deterioration of glucose tolerance, the development of insulin resistance and the consequent damage of the secretory function of beta-cells (Percy-Robby et al., 1982; Swartz et al., 2003; Bernardini et al, 2004). Maintaining the shape weight is therefore a matter of energy intake and expenditure. When for a certain period the energy supply is higher than the expenditure develop overweight and obesity. The incidence of obesity has tripled in the last 20 years and currently in European countries $10-20 \%$ of men and $10-25 \%$ of women are obese (body mass index $>30$ ). There is growing evidence that one of the main trends in obesity is the reduction in physical activity levels. In fact, it seems that in the fight against obesity, the amount of physical activity carried out may be even more decisive than the energy intake. In addition, the tendency to increase incidence of type 2 diabetes has often been attributed to increased obesity, but much evidence shows that inactivity is also a risk factor.

In fact, studies show a $30-50 \%$ reduction in diabetes risk in the most active subjects compared to sedentary subjects (Francavilla et al., 2018). An active lifestyle and at least moderately high level of aerobic activity can also halve the likelihood of cardiovascular disease or dying as a result of them (Francavilla et al., 2013).

It is well known that the physical activity and correct nutrition also promotes weight loss and improves body composition by preserving muscle tissue and increasing fat loss, in particular the reduction of abdominal adiposity. Many data literature reported that the accumulation of visceral adipose tissue is dangerous for health and leads to chronic inflammation status, typical of obese and diabetic subjects (Francavilla et al., 2018; 2013). Indeed, the type 2 diabetes is a worldwide health problem associated with obesity and sedentary lifestyle (Francavilla et al., 2015). Poor glucose and lipid serum profile (High levels of total cholesterol, triglycerides, low-density lipoprotein (LDL) and low col-HDL, or increased fasting glucose and insulin concentration, hypertension, serum adiponectin low levels and increased body mass index (BMI) are significantly associated with increased type 2 diabetes risk and with increased cardiovascular mortality and morbidity in type 2 diabetes patients (Francavilla et al., 2017; 2007; 2018; Valenzano et al., 2019). In addition, the sedentary lifestyle is closely associated to an increased risk of mortality and morbidity for all cause, including dysmetabolic disease. As reported the American Diabetes Association, it is enough to accumulate at least 150 minutes / week (50-70\% of maximum heart rate) and / or intense exercise (> $70 \%$ of maximum heart rate) at least 90 minutes / week, for type 2 diabetes patients, to reduce blood pressure, cardiovascular events, and improve glucose metabolism and insulin sensitivity (Francavilla et al., 2016). In the last decades, a growing number of evidences indicated that 60 minutes of recreational football 2-3 times a week for 12 weeks has positive effects on the cardiovascular, musculoskeletal fitness and on body composition in untrained subjects. These benefits remain for over 6 months even reducing the frequency of training to 60 minutes a week (Polito et al., 2020). In addition, data literature evidenced that 60 minutes of recreational football training twice a week for 24 weeks in men with type 2 diabetes improved $\mathrm{VO}_{2 \text { peak, }}$, cardiopulmonary fitness, resting blood pressure and lowered total body fat mass, and android fat percentage (Di Maio et al., 2020; Corbi et al., 2019). Given these evidences, the aim of this study is to analyse the effects of healthy lifestyle, characterized by a correct diet and by adequate physical activity program in type 2 diabetes patients. 


\section{MATERIAL AND METHODS}

45 patients affected by type 2 diabetes were recruited at school of Engineering, Architecture, and Sport Sciences Enna "KORE" University. All procedures were conformed to the directives of the Declaration of Helsinki and were approved by the Institutional Ethical Committee. All inserted patients had had type II diabetes mellitus for at least two years; patients with associated pathologies were excluded such as: liver disease, nephropathy, heart disease. A basic clinical evaluation was performed and the patients were divided in two different groups.

25 patients in conventional therapeutic and dietary treatment, defined sedentary because they did not carry out any physical activity; 20 patients who was given moderate exercise to perform every day, under the supervision of the sports medicine doctor (EXE patients).

All patients were informed about the benefits of a balanced diet combined with regular physical activity. The standardized diet according to international guidelines.

Both patients groups are monitored at baseline and after six months of protocols. Fasting blood samples were collected at 8:00 am from an antecubital vein, using a 21G Vacutainer blood collection set (BD Diagnostics, Franklin Lakes, NJ, USA). Blood samples were centrifuged and the resultant serum stored at $80^{\circ} \mathrm{C}$, until use. We evaluated biochemical and anthropometric parameters such as glycemia, glycated haemoglobin, total cholesterol, body mass index (BMI), systolic and diastolic blood pressure.

\section{Statistical analysis}

Medians, means $(\mathrm{X})$, standard deviation (SD), standard error (SE), and coefficients of variation (CV) were calculated using standard procedures. The reference limits were calculated both with the parametric method $(X \pm D S)$ and with the non-parametric method (medians, $2.5^{\text {th }}$ and $97.5^{\text {th }}$ percentiles). When the data were "normally" distributed (13) the comparison of the intra-group results was performed with a parametric test: student's $t$ for paired data, otherwise, the comparison was performed with a non-parametric test such as the Wilcoxon test for paired data. In the presence of "normally distributed" data, the comparison between groups (sedentary vs EXE) having homogeneous variances (homoscedastic population), was performed by means of the student test for independent data; when it was not possible to admit the equality a1 = a2 (nonhomogeneous variances), the $t$ test modified by Welch was used. The Snedecor $F$ test was used to check homoscedastic of the populations in comparison. In case of variances with large deviation from the Gaussian curve, a non-parametric test was applied: such as the Wilcoxon test for independent data. The results were processed by the computer scientist with the Compaq Presario433 PC using the software applications Labstat.4 Rel.4.00 Bio-Rad Laboratory 1985.

\section{RESULTS}

In this study we found an amelioration in biochemical and anthropometric parameters such as blood pressure, glycemia, glycated haemoglobin, total cholesterol, body mass index. In particular, we found an improve in blood pressure, glycemia and BMI in both groups of patients sedentary and EXE group as reported in Table $1-10$. 
Table 1. Type 2 diabetes patients - Total population.

\begin{tabular}{lllllllllll}
\hline & $\mathbf{X} \pm$ SD & CV \% & $\begin{array}{l}\mathbf{2 . 5} \\
\text { Perc }\end{array}$ & $\begin{array}{l}\mathbf{9 7 . 5} \\
\text { Perc }\end{array}$ & Median & Val min & $\begin{array}{l}\text { Val } \\
\max \end{array}$ & $\begin{array}{l}\mathbf{2 . 5} \\
\text { Perc }\end{array}$ & $\begin{array}{l}\mathbf{9 7 . 5} \\
\text { Perc }\end{array}$ & $\mathbf{N}$ \\
\hline Age & $64.33 \pm 11.11$ & 17.28 & 42.50 & 86.10 & 65.00 & 44.00 & 86.00 & 44.2 & 85.3 & 45 \\
Weight & $74.40 \pm 20.94$ & 28.14 & 33.40 & 115.40 & 67.00 & 50.00 & 125.00 & 50.50 & 124.0 & - \\
BMI Index & $26.91 \pm 4.94$ & 18.34 & 17.20 & 36.60 & 26.00 & 20.00 & 38.00 & 20.00 & 38.00 & - \\
\hline Female & & & & & & & & & & \\
\hline $\begin{array}{l}\text { Age } \\
\text { Weight }\end{array}$ & $64.88 \pm 11.65$ & 17.96 & 42.05 & 87.72 & 65.00 & 44.00 & 86.00 & - & - & 17 \\
(Kg) & $75.94 \pm 15.81$ & 20.82 & 44.95 & 106.93 & 75.00 & 53.00 & 116.00 & - & - & - \\
BMl & & & & & & & & & & \\
(Index) & $26.59 \pm 4.86$ & 18.28 & 17.06 & 36.12 & 25.00 & 20.00 & 38 & - & - & - \\
\hline Male & & & & & & & & & & \\
\hline $\begin{array}{l}\text { Age } \\
\text { Weight }\end{array}$ & $64.89 \pm 14.79$ & 22.79 & 35.91 & 93.87 & 62.00 & 42.00 & 92.00 & - & - & 28 \\
$\begin{array}{l}\text { BMl } \\
\text { (Index) }\end{array}$ & $26.96 \pm 20.06$ & 26.77 & 35.64 & 114.29 & 69 & 52.00 & 134.00 & - & - & - \\
\hline & & 14.74 & 18.89 & 34.24 & 26.00 & 21.00 & 38.00 & - & - & - \\
\hline
\end{tabular}

Table 2. Sedentary type 2 diabetes patients (TO).

\begin{tabular}{|c|c|c|c|c|c|c|c|c|}
\hline & $X \pm S D$ & CV\% & $\begin{array}{l}2.5 \\
\text { Perc. }\end{array}$ & $\begin{array}{l}97.5 \\
\text { Perc }\end{array}$ & Median & Val min & Val max & $\mathbf{N}$ \\
\hline BMI (Index) & $28.97 \pm 4.80$ & 16.57 & 19.56 & 38.38 & 27.30 & 19.86 & 39.00 & 25 \\
\hline Weight (Kg) & 7.49 & 9.45 & 39.46 & 118.50 & प्र1.00 & 59.00 & 127.00 & 25 \\
\hline $\begin{array}{l}\text { Systolic pressure } \\
(\mathrm{mmHg})\end{array}$ & $140.20 \pm 24.13$ & 17.21 & 92.91 & 187.49 & 135.00 & 105.00 & 190.00 & 25 \\
\hline $\begin{array}{l}\text { Diastolic pressure } \\
\text { (mmHg) }\end{array}$ & $85.48 \pm 7.63$ & 8.93 & 70.52 & 100.44 & 85.00 & 70.00 & 105.00 & 25 \\
\hline $\begin{array}{l}\text { Glycemia (mg/dL) } \\
\text { HBA1C (\%) }\end{array}$ & $\begin{array}{l}135.92 \pm 38.64 \\
8.91 \pm 1.82\end{array}$ & $\begin{array}{l}28.43 \\
20.40\end{array}$ & $\begin{array}{l}60.18 \\
5.35\end{array}$ & $\begin{array}{l}211.66 \\
12.47\end{array}$ & $\begin{array}{l}126.00 \\
8.90\end{array}$ & $\begin{array}{l}92.00 \\
6.30\end{array}$ & $\begin{array}{l}218.00 \\
12.12\end{array}$ & $\begin{array}{l}25 \\
25\end{array}$ \\
\hline $\begin{array}{l}\text { Total cholesterol } \\
(\mathrm{mg} / \mathrm{d} / \mathrm{L})\end{array}$ & $236.17 \pm 33.94$ & 14.37 & 169.64 & 302.70 & 232.00 & 175.00 & 305.00 & 25 \\
\hline
\end{tabular}

Table 3. Sedentary type 2 diabetes patients (T3 months).

\begin{tabular}{|c|c|c|c|c|c|c|c|c|}
\hline & $X \pm S D$ & CV\% & $\begin{array}{l}2.5 \\
\text { Perc. }\end{array}$ & $\begin{array}{l}97.5 \\
\text { Perc }\end{array}$ & Median & Val min & Val max & $\mathbf{N}$ \\
\hline BMI (Index) & & & 18.69 & 35.08 & 2 & 2100 & 3632 & 25 \\
\hline Weigl & & & 40.76 & & & & & 25 \\
\hline $\begin{array}{l}\text { Systolic pressure } \\
(\mathrm{mmHg})\end{array}$ & $129.43 \pm 17.46$ & 13.49 & 95.22 & 163.65 & 126.80 & 105.00 & 160.00 & 25 \\
\hline $\begin{array}{l}\text { Diastolic pressure } \\
(\mathrm{mmHq})\end{array}$ & $83.20 \pm 5.97$ & 7.17 & 71.51 & 94.90 & 80.00 & 75.00 & 100.00 & 25 \\
\hline $\begin{array}{l}\text { Glycemia (mg/dL) } \\
\text { HBA1C }(\%)\end{array}$ & $\begin{array}{l}121.94 \pm 32.13 \\
7.95 \pm 1.26\end{array}$ & $\begin{array}{l}26.35 \\
15.79\end{array}$ & $\begin{array}{l}58.97 \\
5.49\end{array}$ & $\begin{array}{l}184.90 \\
10.42\end{array}$ & & & & $\begin{array}{l}25 \\
25\end{array}$ \\
\hline $\begin{array}{l}\text { Total cholesterol } \\
(\mathrm{mg} / \mathrm{d} / \mathrm{L})\end{array}$ & $219.42 \pm 27.01$ & 12.31 & 166.49 & 272.36 & 215.60 & 175.80 & 289.95 & 25 \\
\hline
\end{tabular}


Table 4. Sedentary type 2 diabetes patients (T6 months).

\begin{tabular}{lllllllll}
\hline & $\mathbf{X} \pm$ SD & CV\% & $\begin{array}{l}\mathbf{2 . 5} \\
\text { Perc. }\end{array}$ & $\begin{array}{l}\mathbf{9 7 . 5} \\
\text { Perc }\end{array}$ & Median & $\begin{array}{l}\text { Val } \\
\text { min }\end{array}$ & $\begin{array}{l}\text { Val } \\
\text { max }\end{array}$ & $\mathbf{N}$ \\
\hline $\begin{array}{l}\text { BMI (Index) } \\
\text { Weight (Kg) }\end{array}$ & $25.27 \pm 3.09$ & 12.23 & 19.22 & 31.33 & 23.90 & 22.00 & 31.60 & 25 \\
$\begin{array}{l}\text { Systolic pressure } \\
\text { (mmHg) }\end{array}$ & $121.45 \pm 12.68$ & 15.57 & 42.20 & 100.71 & 81.50 & 60.00 & 101.00 & 25 \\
$\begin{array}{l}\text { Diastolic pressure } \\
\text { (mmHg) }\end{array}$ & $81.20 \pm 5.78$ & 7.12 & 69.87 & 92.53 & 80.00 & 75.00 & 95.00 & 25 \\
$\begin{array}{l}\text { Glycemia (mg/dL) } \\
\text { HBA1C (\%) }\end{array}$ & $111.10 \pm 24.67$ & 22.22 & 62.71 & 159.49 & 107.00 & 81.58 & 174.70 & 25 \\
$\begin{array}{l}\text { Total cholesterol } \\
\text { (mg/d/L) }\end{array}$ & $7.32 \pm 1.20$ & 16.40 & 4.97 & 9.67 & 7.00 & 5.60 & 10.00 & 25 \\
& $207.53 \pm 18.95$ & 9.13 & 170.40 & 244.67 & 206.80 & 165.80 & 245.12 & 25 \\
\hline
\end{tabular}

Table 5. Sedentary type 2 diabetes patients- comparison of mean.

\begin{tabular}{|c|c|c|c|c|c|c|}
\hline \multicolumn{7}{|c|}{ (T-test or Wilcoxon test) } \\
\hline & \multicolumn{3}{|l|}{ T0 vs T3 months } & \multicolumn{3}{|c|}{ T3 months vs T6 months } \\
\hline & $\begin{array}{l}\text { Differences } \\
\text { between means }\end{array}$ & $\begin{array}{l}\mathrm{t} \\
\text { Student }\end{array}$ & $\mathrm{p}$ & $\begin{array}{l}\text { Differences } \\
\text { between means }\end{array}$ & $\begin{array}{l}\mathrm{t} \\
\text { Student }\end{array}$ & $\mathrm{p}$ \\
\hline BMI (Index) & 2.09 & 8.76 & .00000 & 1.61 & 5.20 & .00003 \\
\hline Weight (Kg) & 5.90 & 5.41 & .00002 & 2.15 & 5.95 & .00001 \\
\hline $\begin{array}{l}\text { Systolic pressure } \\
(\mathrm{mmHg})\end{array}$ & 1.77 & 5.96 & .00000 & 8.25 & 6.56 & .00000 \\
\hline $\begin{array}{l}\text { Diastolic pressure } \\
(\mathrm{mmHg})\end{array}$ & 2.28 & 3.78 & .00091 & 2.00 & 3.48 & .00193 \\
\hline $\begin{array}{l}\text { Glycemia (mg/dL) } \\
\text { HBA1C (\%) }\end{array}$ & $\begin{array}{l}13.98 \\
0.96\end{array}$ & $\begin{array}{l}8.15 \\
5.85\end{array}$ & $\begin{array}{l}.00000 \\
.00000\end{array}$ & $\begin{array}{l}10.84 \\
0.64\end{array}$ & $\begin{array}{l}5.26 \\
5.71\end{array}$ & $\begin{array}{l}.00002 \\
.00001\end{array}$ \\
\hline $\begin{array}{l}\text { Total cholesterol } \\
(\mathrm{mg} / \mathrm{d} / \mathrm{L})\end{array}$ & 16.75 & 6.02 & .00000 & 8.80 & 3.67 & .00024 \\
\hline
\end{tabular}

Table 6. Type 2 diabetes patients EXE groups T0.

\begin{tabular}{|c|c|c|c|c|c|c|c|c|}
\hline & $X \pm S D$ & $\mathrm{CV} \%$ & $\begin{array}{l}2.5 \\
\text { Perc. }\end{array}$ & $\begin{array}{l}97.5 \\
\text { Perc }\end{array}$ & Median & Val min & Val max & $\mathbf{N}$ \\
\hline BMI (Index) & $.94 \pm 3.30$ & 11.83 & 21.46 & 34.41 & 27.79 & 23.00 & 34.28 & 20 \\
\hline Weigh & & & 47.63 & 22.13 & & 9.00 & & 20 \\
\hline $\begin{array}{l}\text { Systolic pressure } \\
(\mathrm{mmHg})\end{array}$ & $141.25 \pm 16.45$ & 11.65 & 109.00 & 173.50 & 137.50 & 120.00 & 185.00 & 20 \\
\hline $\begin{array}{l}\text { Diastolic pressure } \\
(\mathrm{mmHg})\end{array}$ & $89.30 \pm 9.18$ & 10.29 & 71.30 & 107.30 & 88.500 & 75.00 & 105.00 & 0 \\
\hline $\begin{array}{l}\text { Glycemia (mg/dL) } \\
\text { HBA1C }(\%)\end{array}$ & $\begin{array}{l}127.57 \pm 16.40 \\
8.12 \pm 1.26\end{array}$ & $\begin{array}{l}15.25 \\
15.48\end{array}$ & $\begin{array}{l}75.43 \\
5.66\end{array}$ & $\begin{array}{l}139.71 \\
10.58\end{array}$ & $\begin{array}{l}107.02 \\
8.30\end{array}$ & & 8.30 & $\begin{array}{l}20 \\
20\end{array}$ \\
\hline $\begin{array}{l}\text { Total cholesterol } \\
(\mathrm{mg} / \mathrm{d} / \mathrm{L})\end{array}$ & $226.63 \pm 38.44$ & 16.96 & 151.29 & 301.96 & 207.35 & 187.60 & 308.70 & 20 \\
\hline
\end{tabular}


Table 7. Type 2 diabetes patients EXE groups T 3 months.

\begin{tabular}{|c|c|c|c|c|c|c|c|c|}
\hline & $X \pm S D$ & CV\% & $\begin{array}{l}2.5 \\
\text { Perc. }\end{array}$ & $\begin{array}{l}97.5 \\
\text { Perc }\end{array}$ & Median & Val min & Val max & $\mathbf{N}$ \\
\hline BMI (Index) & $2566+290$ & 11.64 & 19.82 & 31.54 & 2520 & 21.70 & 33.00 & 20 \\
\hline Weight (Kg) & & & 47.63 & 14.32 & & & & 20 \\
\hline $\begin{array}{l}\text { Systolic pressure } \\
(\mathrm{mmHg})\end{array}$ & $132.08 \pm 13.60$ & 10.30 & 105.42 & 158.74 & 129.02 & 110.00 & 160.55 & 20 \\
\hline $\begin{array}{l}\text { Diastolic pressure } \\
(\mathrm{mmHg})\end{array}$ & $83.09 \pm 6.19$ & 7.45 & 70.96 & 95.23 & 80.48 & 75.00 & 95.00 & 0 \\
\hline $\begin{array}{l}\text { Glycemia (mg/dL) } \\
\text { HBA1C }(\%)\end{array}$ & $\begin{array}{l}98.14 \pm 15.60 \\
7.16 \pm 1.32\end{array}$ & $\begin{array}{l}15.89 \\
18.37\end{array}$ & $\begin{array}{l}67.57 \\
4.58\end{array}$ & $\begin{array}{l}128.72 \\
9.75\end{array}$ & 6.75 & $\begin{array}{l}62.00 \\
5.60\end{array}$ & $\begin{array}{l}135.40 \\
10.20\end{array}$ & $\begin{array}{l}20 \\
20\end{array}$ \\
\hline $\begin{array}{l}\text { Total cholesterol } \\
(\mathrm{mg} / \mathrm{d} / \mathrm{L})\end{array}$ & $203.74 \pm 24.75$ & 12.15 & 155.24 & 252.25 & 198.20 & 177.60 & 259.60 & 20 \\
\hline
\end{tabular}

Table 8. Type 2 diabetes patients EXE groups T6 months.

\begin{tabular}{|c|c|c|c|c|c|c|c|c|}
\hline & $X \pm S D$ & $\mathrm{CV} \%$ & $\begin{array}{l}2.5 \\
\text { Perc. }\end{array}$ & $\begin{array}{l}97.5 \\
\text { Perc }\end{array}$ & Median & Val min & Val max & $\mathbf{N}$ \\
\hline BMI (Index) & $23.145 \pm 2.41$ & 10.44 & 18.41 & 27.88 & 22.40 & 21.00 & 22.40 & 20 \\
\hline Weight & & & 44.14 & 107.22 & & & & 20 \\
\hline $\begin{array}{l}\text { Systolic pressure } \\
(\mathrm{mmHg})\end{array}$ & $121.64 \pm 12.50$ & 10.28 & 97.14 & 146.14 & 116.40 & 109.00 & 148.00 & 20 \\
\hline $\begin{array}{l}\text { Diastolic pressure } \\
(\mathrm{mmHg})\end{array}$ & $78.46 \pm 6.23$ & 7.95 & 6.24 & 90.68 & 76.91 & 70.00 & 90.00 & 20 \\
\hline Glycemia (mg/dL) & & 12.71 & 68.82 & 114.48 & & 67.00 & 93.50 & 20 \\
\hline HBA1C (\%) & $6.73 \pm 0.79$ & 11.69 & 5.19 & 8.27 & 6.60 & 5.30 & 6.60 & 20 \\
\hline $\begin{array}{l}\text { Total cholesterol } \\
(\mathrm{mg} / \mathrm{d} / \mathrm{L})\end{array}$ & $192.96 \pm 24.37$ & 12.63 & 145.19 & 240.74 & 188.60 & 130.45 & 236.40 & 20 \\
\hline
\end{tabular}

Table 9. Type 2 diabetes patients EXE groups - comparison of mean.

\begin{tabular}{|c|c|c|c|c|c|c|}
\hline \multicolumn{7}{|c|}{ (T-test or Wilcoxon test) } \\
\hline & \multicolumn{3}{|l|}{ T0 vs T3 months } & \multicolumn{3}{|c|}{ T3 months vs T6 months } \\
\hline & $\begin{array}{l}\text { Differences } \\
\text { between means }\end{array}$ & $\begin{array}{l}\mathrm{t} \\
\text { Student }\end{array}$ & $p$ & $\begin{array}{l}\text { Differences } \\
\text { between means }\end{array}$ & $\begin{array}{l}\mathrm{t} \\
\text { Student }\end{array}$ & $p$ \\
\hline BMI (Index) & 2.254 & 9.77 & .000000 & 2.536 & 7.99 & .00000 \\
\hline Weight (Kg) & 5.65 & 8.23 & .00000 & 3.99 & 10.36 & .00000 \\
\hline $\begin{array}{l}\text { Systolic pressure } \\
(\mathrm{mmHg})\end{array}$ & 8.480 & 3.90 & .00010 & 10.39 & 7.87 & .00000 \\
\hline $\begin{array}{l}\text { Diastolic pressure } \\
(\mathrm{mmHg})\end{array}$ & 6.203 & 4.93 & .00009 & 4.633 & 8.40 & .00000 \\
\hline $\begin{array}{l}\text { Glycemia (mg/dL) } \\
\text { HBA1C }(\%)\end{array}$ & $\begin{array}{l}9.427 \\
0.955\end{array}$ & $\begin{array}{l}8.02 \\
5.30\end{array}$ & $\begin{array}{l}.00000 \\
.00004\end{array}$ & $\begin{array}{l}6.490 \\
0.437\end{array}$ & $\begin{array}{l}4.76 \\
2.19\end{array}$ & $\begin{array}{l}.00014 \\
.04157\end{array}$ \\
\hline $\begin{array}{l}\text { Total cholesterol } \\
(\mathrm{mg} / \mathrm{d} / \mathrm{L})\end{array}$ & 4.22 & 5.41 & .00002 & 10.780 & 2.45 & .02393 \\
\hline
\end{tabular}


Table 10. Sedentary type 2 diabetes patients vs type 2 diabetes patients EXE groups-comparison of mean.

\begin{tabular}{|c|c|c|c|c|c|c|c|c|c|}
\hline \multicolumn{10}{|c|}{ Welch test and Wilcoxon test } \\
\hline & \multicolumn{3}{|c|}{ T0 } & \multicolumn{3}{|c|}{ T 3 months } & \multicolumn{3}{|c|}{ T6 months } \\
\hline & $\begin{array}{l}\text { Diff } \\
\text { Means }\end{array}$ & $\begin{array}{l}\mathrm{t} \\
\text { Student }\end{array}$ & $p$ & $\begin{array}{l}\text { Diff } \\
\text { Means }\end{array}$ & $\begin{array}{l}\mathrm{t} \\
\text { Student }\end{array}$ & $P$ & $\begin{array}{l}\text { Diff } \\
\text { Means } \\
\end{array}$ & $\begin{array}{l}\mathrm{t} \\
\text { Student }\end{array}$ & $p$ \\
\hline BMI (Index) & 1.036 & 0.82 & .4160 & 1.205 & 1.085 & 284 & 2.128 & 2.52 & .01543 \\
\hline Weight (Kg) & 14.18 & 9.08 & .00001 & 13.93 & 8.75 & .00001 & 15.77 & 10.91 & .00000 \\
\hline $\begin{array}{l}\text { Systolic } \\
\text { pressure } \\
(\mathrm{mmHg})\end{array}$ & 1.050 & 0.17 & .8689 & 2.646 & 0.5558 & .58122 & 0.452 & 0.1145 & .90940 \\
\hline $\begin{array}{l}\text { Diastolic } \\
\text { pressure } \\
(\mathrm{mmHg})\end{array}$ & 3.818 & 1.523 & .1350 & 0.109 & 0.0595 & .95283 & 2.738 & 1.5247 & 13464 \\
\hline $\begin{array}{l}\text { Glycemia } \\
\text { (mg/dL) }\end{array}$ & 8.32 & 1.95 & 1890 & 23.794 & 3.0336 & .00409 & 19.446 & 3.240 & .00231 \\
\hline HBA1C (\%) & 0.791 & 1.654 & .1054 & 0.790 & 2.0528 & .0462 & 0.591 & 1.987 & .050 \\
\hline $\begin{array}{l}\text { Total } \\
\text { cholesterol } \\
\text { (mg/d/L) }\end{array}$ & 9.549 & 0.884 & .389 & 15.678 & 2.027 & .049 & 14.569 & 2.2573 & .029 \\
\hline
\end{tabular}

\section{DISCUSSION AND CONCLUSIONS}

Obesity is a multifactorial disease and the most primary risk of this is the development of type 2 diabetes. Numerous studies show that the effects of obesity on glucose metabolism result in the deterioration of glucose tolerance, the development of insulin resistance and the consequent damage of the secretory function of beta-cells. The type 2 diabetes is a worldwide health problem associated with obesity and sedentary lifestyle. Our results showed that in type 2 diabetes patients an adequate protocol of physical exercise combined to correct nutrition is able to modify anthropometric and biochemical characteristics. In addition, as show in sedentary groups of type 2 diabetes patients, even alone, correct nutrition can still have beneficial effects that are enhanced by physical activity as show in the EXE patients. A study conducted at the Department of Health and Exercise Science at the University of Tennessee demonstrates how daily walking leads to an improvement in glucose tolerance (Percy-Robby et al., 1982; Bernardini et al., 2004; Francavilla et al., 2018; 2013; 2015). In this study, 18 women with a family history of type II diabetes aged $53.3+/-7$ with a BMl of $35.0+/-5.1 \mathrm{~kg} / \mathrm{m}^{2}$ completed a 4-week period in which they maintained their same habits followed by another 8 week period in which they followed a daily walking schedule where the goal was to reach at least 10,000 steps per day. During the control period the average number of steps was 4972 per day. During the intervention period the participants increased on average the number of steps to 9213 with an average increase of $85 \%$. Although after this period there were no changes in body fat composition, weight and body mass index there were still positive effects both in blood glucose levels after a load after $2 \mathrm{~h}(\mathrm{p}<$ .001) AUC $P=0.0025$, both in the decrease in systolic $(p<.002)$ and diastolic $(p=.002)$ blood pressure. This demonstrates that a minimum level of physical activity still decreases the cardiovascular risk even in those patients who have mobility problems due to overweight or obesity for whom a more demanding program of physical activity cannot be recommended (Valenzano et al., 2019; Francavilla et al., 2017; 2007). On the other hand, also the study of Di Loreto and coll. shows that there is a linear relationship between the benefits that physical activity determines in the diabetic patient and the amount of physical activity performed daily. This relationship occurs starting from $11 \mathrm{METh} /$ week and the maximum benefits are obtained around a value of $27 \mathrm{METh} /$ week. Obviously, if the patient practices a more intense sport, these effects will be 
achieved in a shorter period of time. In a similar 2-year study conducted at the University of Perugia at the Department of Internal Medicine, 182 patients aged $62+/-0.7$ with diabetes of $7.6+/-0.3$ years were recruited. At the beginning, the intervention program consisted of a 30-minute meeting conducted by the doctor in which all the advantages of physical activity were explained to the patient followed a month later by a phone call made by the same doctor to the patient and a 15-minute visit every 3 months for a total of 7 check-ups. Overweight (Defrin et al., 2004) and obese patients were prescribed a diet with a negative caloric balance of $300 \mathrm{Kcal}$ per day, taking into account the energy expenditure associated with voluntary physical activity. Body mass, weight, total and LDL cholesterol, HBA1C indices were assessed at each session. Patients were divided into 6 groups in relation to the share of energy expenditure. At the end of the study, significant reductions were observed in BMI, waist circumference, systolic and diastolic blood pressure, and health care costs related to drugs and diseases. These reductions were the greater the greater the MET. However, the post hoc analysis showed that the minimum energy expenditure for the occurrence of these beneficial effects had to be at least 10 MET (Hill et al., 2005; Di Loreto et al., 2005; Riddell et al., 2006). All this in addition to demonstrating the ineluctability of physical activity shows that it must be considered by the scientific community as a real cornerstone of antidiabetic therapy like diet and oral hypoglycaemic agents. In the past, physical activity has often been attributed a secondary role compared to diet and therapy which has always relegated it to a border-line role.

These and other clinical trials conducted show that physical activity, in addition to having a dramatic role in improving glycometabolic compensation, determines a significant reduction in health expenditure as well as an improvement in the perceived condition of one's health. If this is true in a second type diabetic patient it is equally true in the first type in which it has always been considered in the past if not harmful, certainly not decisive. This can be considered in part true if it occurs sporadically and not planned because in this condition it can more easily disturb the glycometabolic balance. On the contrary, even in the first type diabetic, significant and constant improvements in glycometabolic compensation are obtained through a rhythmic sports therapy program. Data literature reported that the acute effects of physical activity in subjects with insulin-dependent diabetes shows that, although there is an increase in cortisol and Gh levels in In any case, a reduction in glycaemic levels is observed which depends both on the starting glycaemic level and on the duration of the diabetes (Monda et al., 2019). Another study conducted by Bernardini, Vanelli and coll. at the University of Parma on the adherence of physical activity of the youth population with type 1 diabetes shows that there is a long-term decline in HBA1C which correlates with the amount of physical activity (Bernardini et al., 2004). Physical activity has always been considered one of the cornerstones of type 2 diabetes therapy together with diet and pharmacotherapy. However, the results obtained in recent years on the first type diabetic population show that physical activity deserves the same level of consideration as diet and insulin therapy also in the subject with juvenile diabetes by the international scientific community, reaffirming that in any case it must take place in conditions of good glycometabolic compensation and with the consequent insulin adjustment that it requires (Moscatelli et al., 2016; 2015; Patti et al., 2018). The World Health Organization has long recognized that physical and sporting activity plays a fundamental role in promoting positive lifestyle behaviours, capable of promoting health in a global sense and not just as a simple absence of disease. In the context of adopting healthy lifestyles, physical activity plays a fundamental role (Sperandeo et al., 2018; Mazzeo et al., 2013). The protective role of regular physical exercise has been demonstrated above all with regard to cardio-vascular diseases, but also to osteo-articular, metabolic and partly cancerous ones (Messina et al., 2018; 2017; Messina G. et al., 2015). In the healthy population, disease prevention and maintenance of health are based on the identification and control of risk factors and individual behaviours that favour the onset of diseases, as well as on the identification and control of environmental factors that are harmful to health. The project is to promote the knowledge and dissemination among citizens of any age, sex and race of lifestyles for health. Numerous conditions of morbidity, disability and premature mortality can be 
prevented through the adoption of positive, socially shared behavioural models and lifestyle (Messina et al., 2016; Braschi et al., 2011; Polito et al., 2020). According to our experience, in agreement with other research groups in this field, the adoption of a lifestyle has been shown to be able not only to significantly reduce the risk of developing diabetes but to prevent important complications that can be fatal for the patient's life or in any case strongly disabling.

\section{REFERENCES}

Bernardini AL, Vanelli M, Chiari G, lovane B, Gelmetti C, Vitale R, Errico MK. (2004). Adherence to physical activity in young people with type 1 diabetes. Acta Biomed., 75(3):153-7.

Braschi, M.G. Abrignani, V.C.Francavilla, G.Francavilla. (2011). Novel Electrocardiographic Parameters of Altered Repolarization in Uncomplicated Overweight and Obesity Obesity a research journal; 19:4. https://doi.org/10.1038/oby.2010.252

Corbi G, Polito R, Monaco ML, Cacciatore F, Scioli M, Ferrara F. et al. (2019). Adiponectin Expression and Genotypes in Italian People with Severe Obesity Undergone a Hypocaloric Diet and Physical Exercise Program". Nutrients. https://doi.org/10.3390/nu11092195

Defrin R, Josefsberg Z, Karp M.(2004). The effect of acute physical activity on blood glucose levels of children with insulin-dependent diabetes mellitus. Harefuah., 143(12):856-60, 912, 911.

Di Loreto C, Fanelli C, Lucidi P, et al. (2005). Make Your Diabetic Patients Walk. Long-term impact of different amounts of physical activity on type 2 diabetes. Diabetes Care., 28(6):1295-302. https://doi.org/10.2337/diacare.28.6.1295

Di Maio G, Monda V, Messina A, Villano I, Francavilla VC. (2020) Physical activity and modification of lifestyle induce benefits on the health status. Acta Medica Mediterranea., 36(3):1913-1919.

Francavilla VC, Bongiovanni T, Genovesi F, Minafra P, Francavilla G. (2015). Localized bioelectrical impedance analysis: how useful is it in the follow-up of muscle injury? A case report. MED SPORT., 68:323-34.

Francavilla VC, Bongiovanni T, Todaro L, Di Pietro V, Francavilla G. (2017) Probiotic supplements and athletic performance: a review of the literature. MED SPORT., 70:000-000. https://doi.org/10.23736/S0025-7826.17.03037-X

Francavilla C, Bongiovanni T, Todaro L, Genovesi F, Francavilla G. (2016) Risk factors, screening tests and prevention strategies of muscle injuries in elite soccer players: a critical review of the literature. Med Sport., 69: 134-50.

Francavilla G, Francavilla C. Physical exercise is therapy. (2013).MED SPORT., 66:625-8.

Francavilla C, Francavilla A, Braschi M, et al. (2007). Physical exercise and sport activities in patients with and without coronary heart disease. Monadi Arch Chest Dis., 87-95.

Francavilla CV, Sessa F, Salerno M, D' Albano G, Villano I, et al. Influence of Football on Physiological Cardiac Indexes in Professional and Young Athletes. (2018). Front Physiol., 9:153. https://doi.org/10.3389/fphys.2018.00153

Francavilla C, Vitale F, Ciaccio Marcello et al. (2018). Use of saliva in alternative to serum sampling to monitor biomarkers modification in professional soccer players. Frontiers in Phsiology. https://doi.org/10.3389/fphys.2018.01828

Hill JO. (2005). Walking and Type 2 Diabetes Diabetes Care., 28(6):1524-5. https://doi.org/10.2337/diacare.28.6.1524

Mazzeo F, Motti ML, Messina G, et al. (2013). Use of nutritional supplements among south Italian students of Physical Training and Sport University. Current Topics in Toxicology., 9:21-26. 
Messina A, Bitetti I, Precenzano F, lacono D, Messina G, Roccella M, Parisi. et al. (2018). Non-Rapid Eye Movement Sleep Parasomnias and Migraine: A Role of Orexinergic Projections. Front Neurol. 28;9:95. https://doi.org/10.3389/fneur.2018.00095

Messina A, De Fusco C, Monda V, Esposito M, Moscatelli F, Valenzano A, et al. (2016). Role of the orexin system on the hypothalamus-pituitary-thyroid axis. Front. Neural Circuits. 10. https://doi.org/10.3389/fncir.2016.00066

Messina A, Monda V, et al. (2017). Role of the orexin system on arousal, attention, feeding behaviour and sleep disorders. Acta Medica Mediterr., 33(4):645-64. https://doi.org/10.19193/03936384_2017_4_096

Messina G, Zannella C, Monda V, Dato A, Liccardo D, De Blasio S, et al. (2015). The Beneficial Effects of Coffee in Human Nutrition. Biol Med.

Monda V, Villano I, Messina A, Valenzano A, Salerno M, Signorelli S, et al. (2019). Aerobic exercise and orexin A: Role of sympathetic activity and redox system. J Biol Reg and Hom Ag., 33, 587-592.

Moscatelli F, Messina G, Valenzano A, Monda M, et al. (2015). Relationship between RPE and Blood Lactate after Fatiguing Handgrip Exercise in Taekwondo and Sedentary Subjects. Biol Med.

Moscatelli F, Messina G, Valenzano A, Monda V, Viggiano A, Messina A, Petito A, Triggiani Al, Ciliberti MA, Monda M, Capranica L, Cibelli G. (2016). Differences in corticospinal system activity and reaction response between karate athletes and non-athletes. Neurological Sciences., 37(12). https://doi.org/10.1007/s10072-016-2693-8

Patti A, Bianco A, Karsten B, Montalto MA, Battaglia G, et al. (2017). The effects of physical training without equipment on pain perception and balance in the elderly: A randomized controlled trial. Work. 57:23-30. https://doi.org/10.3233/WOR-172539

Percy-Robby W, Broughton P. M. G, Jennings RD, McCormarch JJ, Neill D. W, Saunders R. (1982). A and Warner Mary "Schema consigliato per la valutazione di confenzioni pronte $(<<k i t s>>)$ nel laboratorio di chimica clinica".Giorn it Chim Clin., 7: 219.

Polito R, Francavilla VC, Ambrosi A, Tartaglia N, et al. (2020). The Orexin-A serum levels are strongly modulated by physical activity intervention in diabetes mellitus patients. Journal of Human Sport and Exercise, 15(Proc2), S244-S251. https://doi.org/10.14198/ihse.2020.15.Proc2.15

Polito R, Monda V, Nigro E, Messina A, Di Maio G, Giuliano MT, et al. (2020). The important role of adiponectin and orexin-A, two key proteins improving healthy status: focus on physical activity. Front. Physiol., 11:356. https://doi.org/10.3389/fphys.2020.00356

Riddell MC, et al. (2006). Physical activity, sport, and pediatric diabetes. Pediatr Diabetes. PMID: 16489976 Review. https://doi.org/10.1111/j.1399-543X.2006.00146.X

Sperandeo R, Maldonato MN, Messina A, Cozzolino P, Monda M, Cerroni F, et al. (2018). Orexin system: Network multi-tasking. Acta Medica Mediterr. 34, 349-356. https://doi.org/10.19193/03936384_2018_2 55

Swartz AM, Strath SJ, Bassett DR, Moore JB, Redwine BA, Groer M, Thompson DL. (2003). Increasing daily walking improves glucose tolerance in overweight women. Prev Med., 37(4):356-62. https://doi.org/10.1016/S0091-7435(03)00144-0

Valenzano A, Polito R, Trimigno V, Di Palma A, Moscatelli F, Corso G, Sessa F, Salerno M, Montana A, Di Nunno N, Astuto M, Daniele A, Carotenuto M, Messina G, Cibelli G, Monda V. (2019). Effects of Very Low Calorie Ketogenic Diet on the Orexinergic System, Visceral Adipose Tissue, and ROS Production. Antioxidants., 8(12). https://doi.org/10.3390/antiox8120643

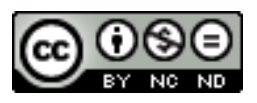

This work is licensed under a Attribution-NonCommercial-NoDerivatives 4.0 International (CC BY-NC-ND 4.0). 